\title{
Geometric potential for plasmon polaritons on curved surfaces
}

\author{
G Della Valle and S Longhi \\ Dipartimento di Fisica, Politecnico di Milano, Piazza L. da Vinci 32, I-20133 Milano, \\ Italy \\ E-mail: giuseppe.dellavalle@polimi.it
}

\begin{abstract}
Surface plasmon polaritons (SPPs) on a curved metal-dielectric interface are shown to experience a geometric potential $V_{\text {top }}$ like quantum particles confined on a curved surface. As opposed to electrons or photons constrained by a squeezing potential, no ambiguities arise in the definition of the geometric potential for SPPs. A general form of $V_{t o p}$ for SPPs on a generalized cylindrical surface is derived by a multiple-scale asymptotic analysis of vectorial Maxwell's equations for the case of a curvature radius larger than the SPP wavelength.
\end{abstract}

PACS numbers: 73.20.Mf, 03.65.Ge, 42.25.-p 
Geometry plays a major role in defining the dynamical properties of quantum particles and classical waves confined on a curved space [1,2]. For instance, it is well known that surface geometry influences the motion of a quantum particle constrained on a curved surface $[1,3,4,5,6,7,8,9]$, like electrons in low-dimensional nanostructures $[10,11,12]$. A similar behavior occurs for optical waves squeezed on a curved thin dielectric guiding layer [13]. As in the 'classical' (e.g. newtonian or ray-optics) limits the motion is ruled by the surface metric and the force-free trajectories are geodesics, in the full wave regime an additional frictional potential $V_{t o p}$, referred to as the geometric potential, is found $[3,4,5,6]$. Though $V_{\text {top }}$ vanishes in the classical limit (as it should), its dependence on the surface topology has been a matter of great controversies which have been especially discussed in the quantum mechanical context $[4,7,8,9]$. An accepted approach, originally proposed by Jensen, Koppe and da Costa [5, 6], is to squeeze the quantum particle on the surface by a physical confining potential. This approach has been recently adopted for confined optical waves as well [13]. Even in this case some ambiguities remain due to the freedom in selecting the squeezing potential [9]. Such difficulties cast a shadow over the physical relevance of any geometric potential for three-dimensional particles or waves constrained on a surface, because the way they sense the surrounding space strongly depends on the squeezing potential. Some authors also argued that ideal squeezing procedures generally correspond to unrealistic restrictions $[7]$.

In this Letter we show that a geometric potential, which does not suffer from any ambiguity, does exist at a curved metal-dielectric interface for surface plasmon polaritons (SPPs), i.e. for coupled modes of plasmons and photons. As compared to electrons or photons squeezed on a surface, SPPs are intrinsically two-dimensional quasi particles and do not require any squeezing potential. SPPs have attracted special attention in recent years for their relevance in subwavelength optics and nanophotonics $[14,15]$. In spite of the considerable efforts devoted to study SPPs in different curved geometries [16, 17, 18, 19, 20, 21], including SPPs scattering and radiation at bends or interfaces $[22,23,24,25]$, the concept and properties of a geometric potential for SPPs has been overlooked.

Here we consider SPPs on a generalized cylindrical surface [Fig. 1(a)] and derive, by a multiple scale asymptotic analysis of full vectorial Maxwell's equations, a general form of $V_{t o p}$. The predictions of the asymptotic analysis, such as the appearance of bound states sustained by the geometric potential, are confirmed by full-vectorial numerical simulations in curved Ag-glass interfaces. Let us consider SPPs at optical frequency $\omega=2 \pi c_{0} / \lambda$ on a curved metal-dielectric interface defined by a generalized cylindrical surface and let us introduce a set of local curvilinear coordinates $(\sigma, \eta, z)$ around the surface, as shown in Fig. 1(a). If we normalize the spatial variables to the reduced wavelength $\lambda=\lambda /(2 \pi)=1 / k$ and indicate by $\mathbf{u}=\left(c_{0} B_{\sigma}, E_{\eta}, E_{z}\right)$ and $\mathbf{v}=\left(E_{\sigma}, c_{0} B_{\eta}, c_{0} B_{z}\right)$ the mixed components of electric $\mathbf{E}$ and magnetic $\mathbf{B}$ fields, Maxwell's equations in the curvilinear coordinate system can be cast in the following 
(a)

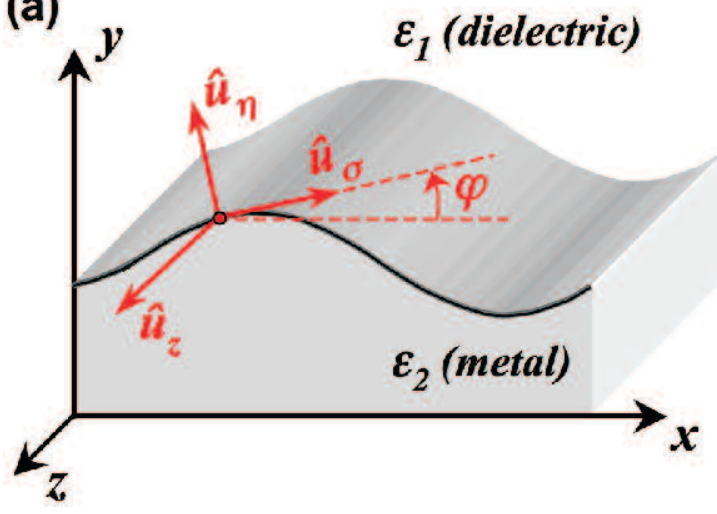

(b)

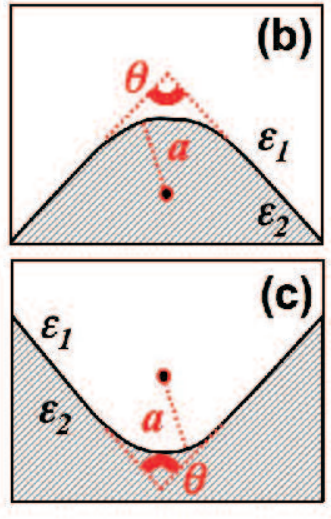

Figure 1. (color online) (a) Schematic of a generalized-cylinder (i.e. $z$-invariant) metal-dielectric interface and local curvilinear coordinate system. (b) and (c): sketch of convex and concave 'bookcover' metal-dielectric interfaces.

form

$$
\mathcal{L}_{T M} \mathbf{u}=\mathbf{g}_{T M}, \mathcal{L}_{T E} \mathbf{v}=\mathbf{g}_{T E}
$$

where we have set

$$
\mathcal{L}_{\mathrm{TM}}=\left(\begin{array}{ccc}
i & -\partial_{z} & \partial_{\eta} \\
\partial_{z} & -i \epsilon & 0 \\
\partial_{\eta} & 0 & i \epsilon
\end{array}\right), \mathcal{L}_{\mathrm{TE}}=\left(\begin{array}{ccc}
\partial_{z} & i & 0 \\
-\partial_{\eta} & 0 & i \\
-i \epsilon & -\partial_{z} & \partial_{\eta}
\end{array}\right),
$$

$\epsilon$ is the relative dielectric permittivity $\left[\epsilon=\epsilon_{1}\right.$ in the dielectric, at $\eta>0 ; \epsilon=\epsilon_{2}$ in the metal, at $\eta<0]$, and

$$
\mathbf{g}_{\mathrm{TM}}=\left(\begin{array}{c}
0 \\
\frac{\partial_{\sigma} v_{3}}{h_{1}} \\
\frac{\partial_{\sigma} v_{2}}{h_{1}}+\frac{u_{1}}{R h_{1}}
\end{array}\right), \mathbf{g}_{\mathrm{TE}}=\left(\begin{array}{c}
\frac{\partial_{\sigma} u_{3}}{h_{1}} \\
-\frac{\partial_{\sigma} u_{2}}{h_{1}}-\frac{v_{1}}{R h_{1}} \\
0
\end{array}\right) .
$$

In the above equations, $h_{1}=1-\eta / R, R=(d \varphi / d \sigma)^{-1}$ is the local radius of curvature of the cylinder line - normalized to $\lambda$ and with its appropriate sign - and $\varphi(\sigma)$ is the angle defined in Fig. 1(a). Pure TM-polarized SPP modes, corresponding to $\mathbf{v}=0$ in Eq. (1), exist solely for a constant curvature $1 / R$, i.e. for a circular cylinder, and for axially symmetric fields $[16,17,18]$. For a general cylindrical surface (not necessarily closed), mixed TE and TM modes are instead found. However, for a sufficiently smooth surface with a local radius of curvature much larger than the photon wavelength, i.e. for $|R| \gg 1$, we may look for a solution to Eq. (1) as an asymptotic expansion in a smallness parameter $\alpha$ such that at leading order the TM-polarized SPP mode propagating along the $z$ direction of the locally-flat interface is retrieved. The structure of Eq. (1) suggests to assume $R \sim 1 / \alpha^{2}$, a slow dependence of the fields with respect to $\sigma$ on the scale $\sigma_{1}=\alpha \sigma$, and to introduce the following power series expansions $\mathbf{u}=\mathbf{u}^{(0)}+\alpha^{2} \mathbf{u}^{(2)}+\alpha^{4} \mathbf{u}^{(4)}+\ldots$ and $\mathbf{v}=\alpha \mathbf{v}^{(1)}+\alpha^{3} \mathbf{v}^{(3)}+\ldots$ for TM and TE field components, respectively. To remove secular growing terms that arise in the asymptotic analysis, multiple scales $z_{0}=z, z_{1}=\alpha z, z_{2}=\alpha^{2} z, \ldots$ are then introduced for the spatial 
variable $z$. By substituting the power expansions for $\mathbf{u}$ and $\mathbf{v}$ into Eq. (1) and using the derivative rules $\partial_{z}=\partial_{z_{0}}+\alpha \partial_{z_{1}}+\alpha^{2} \partial_{z_{2}}+\ldots$ and $\partial_{\sigma}=\alpha \partial_{\sigma_{1}}$, a hierarchy of equations for successive corrections to $\mathbf{u}$ and $\mathbf{v}$ is obtained. At leading order $\left(\sim \alpha^{0}\right)$ one retrieves the usual TM-polarized SPP mode on the locally-flat planar interface propagating along the $z$ direction, namely

$$
\mathbf{u}^{(0)} \equiv\left(\begin{array}{c}
c_{0} B_{\sigma}^{(0)} \\
E_{\eta}^{(0)} \\
E_{z}^{(0)}
\end{array}\right)=F\left(\begin{array}{c}
1 \\
-n_{e} / \epsilon \\
-i \gamma / \epsilon
\end{array}\right) \exp \left(-i n_{e} z_{0}-\gamma \eta\right)
$$

In Eq. (4), the amplitude $F$ depends on slow spatial variables $\sigma_{1}, z_{1}, z_{2}, \ldots ; n_{e}=$ $\left[\epsilon_{1} \epsilon_{2} /\left(\epsilon_{1}+\epsilon_{2}\right)\right]^{1 / 2}$ is the effective index of the SPP; and $\gamma=\gamma_{1}$ for $\eta>0, \gamma=-\gamma_{2}$ for $\eta<0$ are the decay constants of the field tails, normalized to $k$, in the dielectric and metal regions, respectively. Note that $\gamma_{1} / \epsilon_{1}=-\gamma_{2} / \epsilon_{2}$, i.e. the ratio $\gamma / \epsilon$ is continuous at the interface. In order to satisfy at the interface $\eta=0$ the field boundary conditions at successive orders in the asymptotic analysis, a power expansion $\gamma_{1,2}=\gamma_{1,2}^{(0)}+\alpha \gamma_{1,2}^{(1)}+\alpha^{2} \gamma_{1,2}^{(2)}+\ldots$ is assumed, where $\gamma_{1,2}^{(k)}$ are generally allowed to vary on the slow spatial scale $\sigma_{1}$. The leading order terms are independent of $\sigma_{1}$ and are given by the well-known relations for SPPs at a flat interface

$$
\gamma_{1,2}^{(0)}=\left[-\epsilon_{1,2}^{2} /\left(\epsilon_{1}+\epsilon_{2}\right)\right]^{1 / 2}
$$

For instance, for a metal with a dielectric function approximated by the simplest Drude relation $[16,17,18] \epsilon_{2}(\omega) \simeq 1-\left(\omega_{p} / \omega\right)^{2}$, where $\omega_{p}$ is the bulk plasma frequency, one has $\epsilon_{2} \sim \operatorname{Re}\left(\epsilon_{2}\right)<0, n_{e} \sim \operatorname{Re}\left(n_{e}\right)$ and the existence of SPPs requires $\omega<\omega_{p} /\left(1+\epsilon_{1}\right)^{1 / 2}$. The evolution of the amplitude $F$ on the slow spatial scales $z_{1}, z_{2}, \ldots$ is derived as a solvability condition at orders $\sim \alpha, \alpha^{2}, \ldots$ in the asymptotic expansion. At order $\sim \alpha$, one obtains $\partial_{z_{1}} F=0, \gamma_{1}^{(1)}=\gamma_{2}^{(1)}=0$, and the following TE-polarized correction $\mathbf{v}^{(1)}$ to the fields is found

$$
\left(\begin{array}{c}
E_{\sigma}^{(1)} \\
c_{0} B_{\eta}^{(1)} \\
c_{0} B_{z}^{(1)}
\end{array}\right)=\frac{\partial F}{\partial \sigma_{1}}\left(\begin{array}{c}
\left(-\epsilon_{1} \epsilon_{2}\right)^{-1 / 2} \\
0 \\
-i / n_{e}
\end{array}\right) \exp \left(-i n_{e} z_{0}-\gamma \eta\right) .
$$

At order $\sim \alpha^{2}$, the solvability condition yields

$$
2 n_{e} \frac{\partial F}{\partial z_{2}}+i \frac{\partial^{2} F}{\partial \sigma_{1}^{2}}-i \frac{n_{e}^{2}}{R} \frac{\gamma}{\epsilon} F=0
$$

and the following corrections to the decay constants $\gamma_{1}$ and $\gamma_{2}$ are obtained from the field boundary conditions

$$
\gamma_{1,2}^{(2)}=\frac{2 \epsilon_{2,1}^{2}-\epsilon_{1,2}^{2}-\epsilon_{1} \epsilon_{2}}{2 R\left(\epsilon_{1}^{2}-\epsilon_{2}^{2}\right)} .
$$

If we stop the asymptotic analysis at this order and re-introduce the non-scaled physical spatial variables $z, \sigma$ and $R=(d \varphi / d \sigma)^{-1}$, the following evolution equation for the SPP envelope $F$ along the propagation direction $z$ is finally obtained

$$
-i \lambda \frac{\partial F}{\partial z}=-\frac{\lambda^{2}}{2 n_{e}} \frac{\partial^{2} F}{\partial \sigma^{2}}+V_{t o p}(\sigma) F
$$


where the geometric potential $V_{\text {top }}$ is given by

$$
V_{\text {top }}(\sigma)=\frac{\lambda n_{e}}{2 R} \sqrt{-\frac{1}{\epsilon_{1}+\epsilon_{2}}}
$$

Equation (9) shows that a SPP that diffracts on the surface as it propagates along the $z$ direction is subjected to a frictional potential $V_{\text {top }}$ which vanishes in the ray-optics limit, i.e. for $\lambda \rightarrow 0$. This potential plays a similar role to the geometric potential experienced by electrons constrained on a surface by a confining potential [6] or by photons squeezed on a thin curved dielectric layer [13], and may lead to the existence of SPPs bound states, i.e. geometric-induced SPP wave guiding. As opposed to truly three-dimensional particles (e.g. electrons or photons), the definition of $V_{t o p}$ for SPPs given here is well-posed since it does not require any squeezing procedure, which is at the heart of controversies [7, 9]. Additionally, the dependence of the geometric potential on the extrinsic curvature $\kappa=1 /(2 R)$ of the surface differs substantially in the two cases. For two-dimensional quasi-particles like SPPs, according to Eq. (10) $V_{\text {top }}$ turns out to be proportional to $\kappa$, and is thus sensitive to the magnitude and sign of the curvature. This means that a SPP on a convex or on a concave metal-dielectric interface, such as those shown in Figs. 1(b) and 1(c), behaves differently: as $V_{\text {top }}$ produces a potential well in case of Fig. 1(b), it transforms into a potential barrier in the other case [Fig. 1(c)]. Conversely, for three-dimensional particles like electrons or photons squeezed on the same surface by a confining potential, $V_{\text {top }}$ turns out to be proportional to the square of the extrinsic curvature $\kappa[6,13]$. In the optical context, the geometric potential defined by Eq. (10) corresponds to a local (i.e. $\sigma$-dependent) correction to the effective index of the SPP wave due to surface curvature. The dependence of $R$ on $\sigma$ thus provides the guiding or anti-guiding properties of the interface.

Extensive numerical simulations of Eq. (1), based on standard finite element methods (FEM), have confirmed the validity of the asymptotic analysis and the correctness of Eq. (10) for various shapes of the cylinder line. As a first example, we investigated the formation of bound modes sustained by an open cylindrical Ag-glass interface simulating a square potential [Fig. 1(b)]. This interface is analogous to the 'bookcover' surface discussed by da Costa [6] and consists of a plane bent around the surface of a circular cylinder of radius $a$ and angular aperture $\theta$. The corresponding geometric potential is a finite square well, which is expected to support localized SPP modes, denoted by $\mathrm{SPP}_{i}$, analogous to the quantum-mechanical eigenmodes of a square potential well. FEM numerical simulations confirmed the existence of bound SPP modes, as shown in Fig. 2. In the simulations, a real-valued $\epsilon_{2}$ has been assumed for simplicity, and its dependence on $\lambda$ has been taken from the refractive index experimental data reported in [26]. At $450 \mathrm{~nm}$ wavelength, the well supports two bound states $\mathrm{SPP}_{1}$ and $\mathrm{SPP}_{2}$ solely, with effective indices $n_{i}=n_{e}+\Delta n_{i}$ that differ from the effective index $n_{e}$ of a flat SPP mode because of the contributions $\Delta n_{i}$ arising from the geometric potential (see Fig. 2(d)).

The numerically-computed distributions of the magnetic-field amplitude for the 
G Della Valle et al., Geometric potential for plasmon polaritons on curved surfaces...6
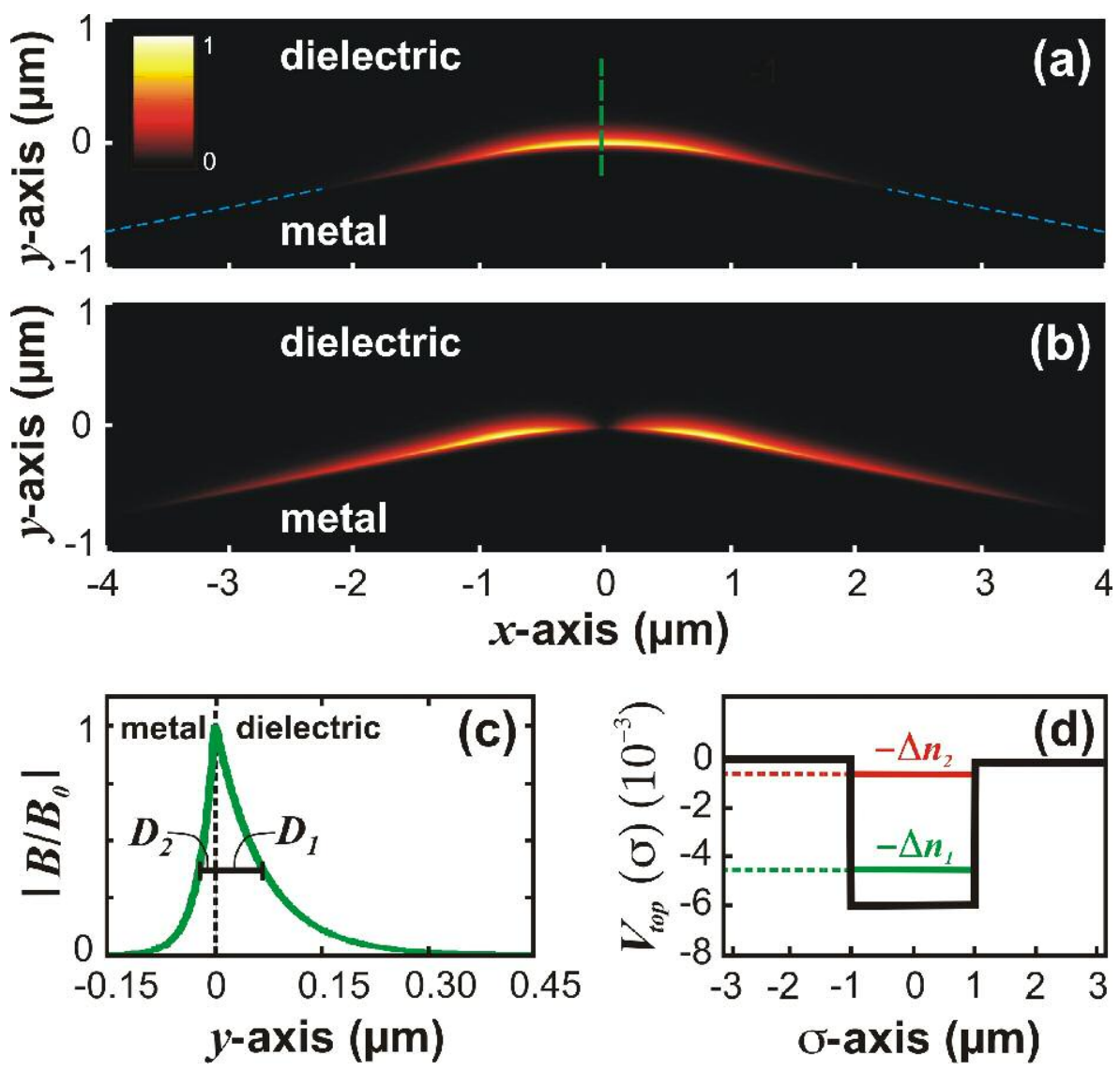

Figure 2. (color online) FEM numerically-computed distribution of normalized magnetic field amplitude at $450 \mathrm{~nm}$ for (a) the fundamental $\mathrm{SPP}_{1}$ mode, and (b) the second-order $\mathrm{SPP}_{2}$ mode in a convex structure with $a=5 \mu \mathrm{m}, \theta=157^{\circ}$ and $\epsilon_{1}=2.25$. (c) Cross section profile of the $\mathrm{SPP}_{1}$ mode at $x=0$. (d) Geometric potential of the convex structure as given by Eq. (10) and its two eigenvalues, corresponding to the corrections $\Delta n_{1}$ [for the eigenmode $\mathrm{SPP}_{1}$ of Fig. 2(a)] and $\Delta n_{2}$ [for the eigenmode $\mathrm{SPP}_{2}$ of Fig. 2(b)] to the effective index $n_{e}$.

fundamental and first-order SPP modes are depicted in Figs. 2(a) and 2(b), respectively. The field profile across the metal-dielectric interface exhibits a typical asymmetric exponential localization, as shown in Fig. 2(c), with a 1/e decay length $D_{1} \sim 68 \mathrm{~nm}$ in the dielectric and $D_{2} \sim 22 \mathrm{~nm}$ in the metal, in excellent agreement with the theoretical values $\lambda / \gamma_{1}^{(0)}=69.3 \mathrm{~nm}$ and $\lambda / \gamma_{2}^{(0)}=22.3 \mathrm{~nm}$ predicted by Eq. (5). The numericallycomputed behavior of the effective index shift $\Delta n_{1}$ versus $\lambda$ (dispersion curve) for the fundamental $\mathrm{SPP}_{1}$ mode is depicted in Fig. 3(a). Similarly, Fig. 3(b) shows the behavior of $\Delta n_{1}$ as a function of the radius of curvature $a$ for a fixed wavelength. The results of FEM simulations (points) are compared to the theoretical predictions $\ddagger$ based on Eq. (10) (solid curves). Note the excellent agreement between the two results, even for sharplycurved interfaces with a radius $a$ comparable to $\lambda$. Finally, the FEM analysis confirmed

$\ddagger$ In the framework of the amplitude equation (9), the modal profiles $F_{i}$ and corresponding corrections $\Delta n_{i}$ to the effective index are computed from the eigenvalue problem $\mathcal{H} F_{i}=-\Delta n_{i} F_{i}$ with Hamiltonian $\mathcal{H}=-\star^{2} /\left(2 n_{e}\right) \partial_{\sigma}^{2}+V_{\text {top }}(\sigma)$. 
that for the corresponding concave metal-dielectric interface of Fig. 2, leading to a potential barrier, SPP bound modes do not exist for the entire radius range $0.5-20 \mu \mathrm{m}$ considered above.
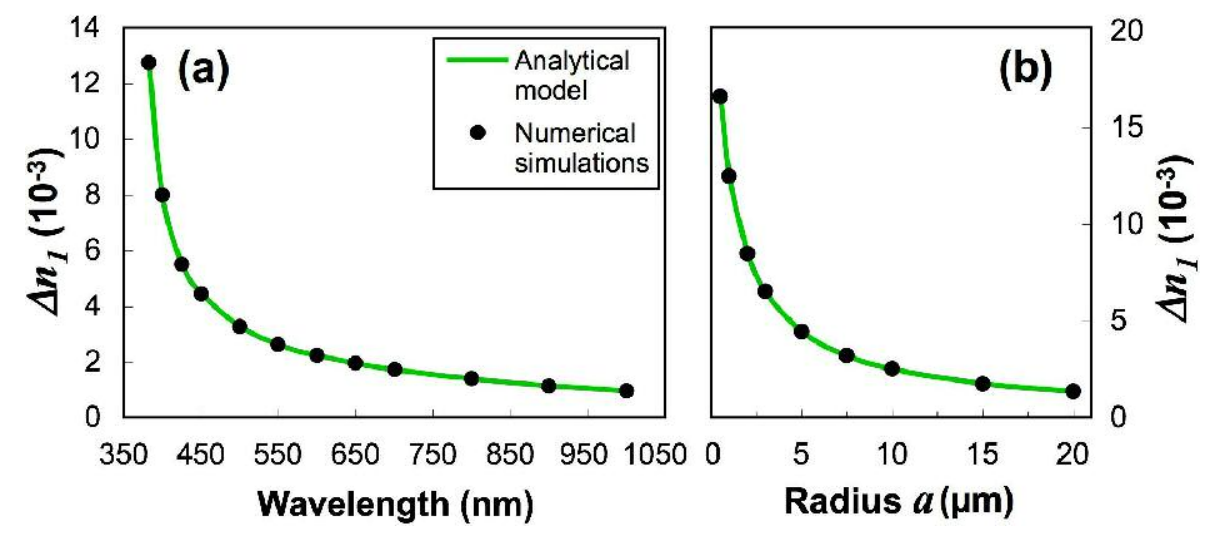

Figure 3. (color online) (a) Dispersion curve for the $\mathrm{SPP}_{1}$ mode (other parameter values as in Fig. 2). (b) Behavior of the effective index shift $\Delta n_{1}$ versus radius $a$ for the silver-glass convex structure of Fig. 1 (b) for $\lambda=450 \mathrm{~nm}$ and $\theta=157^{\circ}$. Circles: FEM numerical results; solid curves: asymptotic analysis results.

As a second example, we investigated a closed elliptical Ag-glass interface in either a convex or concave geometry (Fig. 4). In both cases, FEM simulations show the existence of a set of SPP modes with increasing number of nodes along the ellipse. In particular, a doublet of nearly-degenerate modes, with the largest effective indices and showing an almost coincident distribution of $|\mathbf{B}|$, is found. For the convex geometry, corresponding to a cylindrical silver rod embedded in glass [inset in Fig. 4(a)], the doublet [left panel in Fig. 4(a)] is localized around the two points of the ellipse where the local curvature $|\kappa|$ is the largest. On the contrary, the doublet of the concave structure, corresponding to a cylindrical glass hole embedded in silver [inset in Fig. 4(a)], is localized at the minima of the curvature $|\kappa|$. This behavior is in agreement with the shapes of the geometric potential in the two cases, which are shown in the right panels of Fig. 4(a) and (b). While the convex structure corresponds to a negative curvature, i.e. to $V_{\text {top }}<0$, the concave structure results in a positive $V_{\text {top }}$ because of its positive curvature. In both cases, the geometric potential is a double well on a closed loop, and the nearly-degenerate doublet modes found in FEM simulations are just the symmetric and anti-symmetric modes of a quantum-mechanical double well.

In the previous analysis, we neglected absorption losses in the metal, i.e. the imaginary part of $\epsilon_{2}$. If $\operatorname{Im}\left(\epsilon_{2}\right)$ is much smaller than $\operatorname{Re}\left(\epsilon_{2}\right), \operatorname{Im}\left(\epsilon_{2}\right)$ enters in the asymptotic analysis of Eq. (1) as a perturbation term. At leading order, one can show that its effect is just to introduce an exponential damping along $z$ of the envelope $\psi$ like for a plane $\mathrm{SPP}$, which results in a finite propagation length $L_{p}$. For instance, for $\mathrm{Ag}$ at $\lambda=1 \mu \mathrm{m}$ and for the convex geometry of Fig. 2, one has $L_{p} \simeq\left[\operatorname{Re}\left(\epsilon_{2}\right)\right]^{2} /\left[\operatorname{Im}\left(\epsilon_{2}\right) n_{e}^{3} k\right] \simeq 198.1 \mu \mathrm{m}$ for the plane SPP. A full numerical analysis of Eqs.(1), which includes metal absorption, 


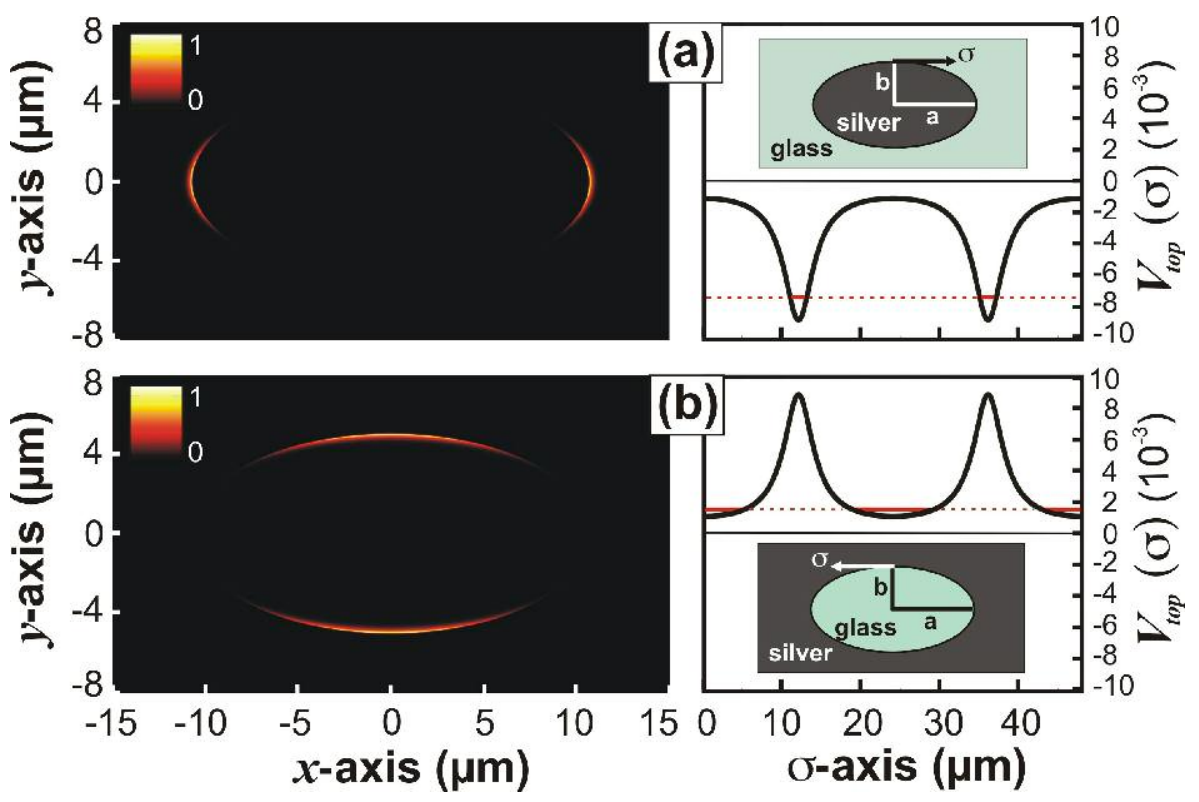

Figure 4. (color online) (a) Left panel: fundamental mode (normalized amplitude of the magnetic field) supported by an elliptical silver rod in glass for $\lambda=550 \mathrm{~nm}$, $a=10 \mu \mathrm{m}$, and $b=5 \mu \mathrm{m}$ (see inset in the right panel). Right panel: topological potential as a function of the curvilinear coordinate $\sigma$. The horizontal red lines represent the nearly-degenerate doublet of the double-well potential on a closed loop. (b) Same as (a) for the complementary (i.e. concave) structure: an elliptical glass hole in silver.

yields $L_{p} \simeq 193.5 \mu \mathrm{m}$, which is in excellent agreement with the predicted value. As $L_{p}$ is (about two times) larger than the characteristic diffractive length of the mode, guiding or anti-guiding of convex/concave surfaces should be therefore observable in realistic experimental conditions.

It is worth finally noting that the geometric potential induced by an open curved surface of concave geometry [Fig. 1(c)] does not sustain SPP modes, which are conversely found in small-angle V-grooves, also called Channel Plasmon Polaritons (CPPs) (see, for instance, [28] and references therein). Actually, there is no contradiction between our analysis and the well known result that V-grooves sustain CPPs. In fact, the guidance properties of small-angle $\mathrm{V}$-grooves are governed by the interaction between the two SPP waves localized at the metal-insulator-metal double interface, which can be captured by using more common effective index methods [29]. On the contrary, in our structures large radii of curvature and sufficiently large aperture angles $\theta$ are assumed, thus preventing any interaction between SPP modes at the opposite sides of the concave structure.

In conclusion, SPPs on a curved metal-dielectric interface experience a frictional potential of geometric nature like quantum particles confined on a curved surface. However, the form of the geometric potential for SPPs is very different owing to 
the intrinsic two-dimensional nature of SPPs. Most importantly, the SPPs geometric potential does not suffer from any ambiguity that arises from the need of a squeezing potential as in other systems $[4,7,8,9]$. Our results suggest that plasmonics may provide an accessible laboratory system to test on the nanoscale the signatures of geometric potentials in a curved space.

\section{References}

[1] Sniatycki J 1980 Geometric Quantization and Quantum Mechanics (Springer, New York)

[2] Leonhardt U and Philbin T G 2009 Progress in Optics 5369 (edited by Emil Wolf)

[3] Dewitt B S 1957 Rev. Mod. Phys. 293774

[4] Schulman L S 1981 Techniques and Applications of Path Integration (Wiley, New York).

[5] Jensen H and Koppe H 1971 Ann. Phys. (N.Y.) 63586

[6] da Costa R C T 1981 Phys. Rev. A 231982

[7] van Kampen N G and Lodder J J 1984 Am. J. Phys. 52419

[8] Ikegami M, Nagaoka Y, Takagi S and Tanzawa T 1992 Prog. Theor. Phys. 88229

[9] Kaplan L, Maitra L T and Heller E J 1997 Phys. Rev. A 562592

[10] Cantele G, Ninno D and Iadonisi G 2000 Phys. Rev. B 6113730

[11] Marchi A, Reggiani S, Rudan M and Bertoni A 2005 Phys. Rev. B 72035403

[12] Ferrari G and Cuoghi G 2008 Phys. Rev. Lett. 100230403

[13] Longhi S 2007 Opt. Lett. 32 2647; Batz S and Peschel U 2008 Phys. Rev. A 78043821

[14] Barnes W L, Dereux A and Ebbesen T W 2003 Nature (London) 424824

[15] Ebbesen T, Genet C and Bozhevolnyi S I 2008 Phys. Today 6144

[16] Pfeiffer C A, Economou E N and Ngai K L 1974 Phys. Rev. B 103038

[17] Vasconcelos E F, de Oliveira N T and Farias G A 1991 Phys. Rev. B 4413740

[18] Takahara J, Yamagishi S, Takai H, Morimoto A and Kobayashi T 1997 Opt. Lett. 22475

[19] Stockman M I 2004 Phys. Rev. Lett. 93137404

[20] Smolyaninov I I, Balzano Q and Davis C C 2005 Phys. Rev. B 72165412

[21] Passian A, Ritchie R H, Lereu A L, Thundat T and Ferrell T L 2005 Phys. Rev. B 71115425

[22] Veronis G and Fan S 2005 Appl. Phys. Lett. 87131102

[23] Radko I P, Søndergaard T and Bozhevolnyi S I 2006 Opt. Express 144107

[24] Hasegawa K, Nöeckel J U and Deutsch M 2004 Appl. Phys. Lett. 841835

[25] Hasegawa K, Nöckel J U and Deutsch M 2007 Phys. Rev. A 75063816

[26] Johnson P B and Christy R W 1972 Phys. Rev. B 64370

[27] Noginov M A, Podolskiy V A, Zhu G, Mayy M, Bahoura M, Adegoke J A, Ritzo B A, Reynolds K 2008 Opt. Express 161385

[28] Bozhevolnyi S I, Volkov V S, Devaux E, and Ebbesen T W 2005 Phys. Rev. Lett. 95046802

[29] Bozhevolnyi S I, Nerkararyan K V 2009 Opt. Lett. 342039 